\title{
Philosophiques
}

\section{Spinoza et le problème de l'akrasia : Un aspect négligé de l'ordo geometricus}

\section{Jacques-Henri Gagnon}

Volume 29, numéro 1, printemps 2002

Spinoza sous le prisme de son anthropologie

URI : https://id.erudit.org/iderudit/009563ar

DOI : https://doi.org/10.7202/009563ar

Aller au sommaire du numéro

Éditeur(s)

Société de philosophie du Québec

ISSN

0316-2923 (imprimé)

1492-1391 (numérique)

Découvrir la revue

Citer cet article

Gagnon, J.-H. (2002). Spinoza et le problème de l'akrasia : Un aspect négligé de l'ordo geometricus. Philosophiques, 29(1), 57-71.

https://doi.org/10.7202/009563ar d'utilisation que vous pouvez consulter en ligne.

https://apropos.erudit.org/fr/usagers/politique-dutilisation/ 


\title{
Spinoza et le problème de l'akrasia : Un aspect négligé de l'ordo geometricus
}

\author{
JACQUES-HENRI GAGNON \\ Ministère des Affaires étrangères et du commerce international du Canada \\ Jacques-Henri-Gagnon@wanadoo.fr
}

\begin{abstract}
RÉSUMÉ. - Dans un premier temps, ce texte expose de façon succincte comment l'Éthique traite de la problématique de l'akrasia, c'est-à-dire de la faiblesse de la volonté. Traditionnellement exprimée par la sentence « voir le meilleur et pourtant faire le pire », cette problématique incontournable pour toute éthique, est développée dans la quatrième partie de l'Éthique. Dans un deuxième temps, nous montrons comment l'ordre géométrique utilisé par Spinoza pour écrire son livre constitue un aspect important de la solution spinoziste au problème de l'akrasia.
\end{abstract}

ABSTRACT. - The question of the weakness of the will, traditionally named akrasia after Aristotle's Nicomachean Ethics (Book III), is tackled in part 4 of Spinoza's Ethics. After a brief presentation of this problematic in the Ethics, the author shows how the geometrical order chosen by Spinoza to write his book constitutes a great part of the strategy put in place to concretely resolve this question.

\section{À Hélène}

Rechercher dans les livres ce qu'est l'homme, je le devrais tenir pour un plus mauvais travail que s'en assurer soi-même, puisque bien peu sont aptes à mettre dans un livre l'homme tel qu'il est. Lichtenberg Sudelbücher

Il n'est pas une seule pensée importante dont la bêtise ne sache aussitôt

faire usage; elle peut se mouvoir dans toutes les directions et prendre tous les costumes de la vérité. La vérité, elle, n'a jamais qu'un seul vêtement, un seul chemin : elle est toujours handicapée. La bêtise dont il s'agit là n'est

pas une maladie mentale; ce n'en est pas moins la plus dangereuse des maladies de l'esprit, parce que c'est la vie même qu'elle menace.

Robert Musil Über das Dummheit

L'écriture d'une éthique pose des problèmes spécifiques liés à la nature propre qu'entretient son contenu avec l'action. Car si un tel livre veut être autre chose qu'un exercice en vanité, ou qu'une expression du ressentiment de l'auteur excité par le comportement jugé coupable de ses semblables, il doit trouver les moyens de sortir de l'état dans lequel il semble immanquablement condamné : celui de lettre morte.

Cette question, aussi fondamentale que négligée par nos éthiciens et moralistes modernes (ou postmodernes !), marqués comme ils le sont par l'avidité de découvrir une solution miracle, et dont le miraculeux tiendrait tout entier dans la rapidité de son efficacité et la facilité de son administration, cette question donc fut l'objet d'une réflexion soutenue chez les philosophes de 
l'Antiquité. La chose est bien connue, et les travaux de Pierre Hadot et de quelques autres y sont assurément pour beaucoup dans la compréhension renouvelée que nous avons aujourd'hui des pratiques philosophiques des anciens.

Cependant, il semble bien que, si on veut croire le tableau scolaire et institutionnalisé de l'histoire de la philosophie - miroir que la philosophie se donne à elle-même pour ne pas voir un monde qui correspond trop peu à son monde des idées, et dans lequel elle se repaît par la contemplation de sa glorieuse Histoire - il semble bien donc, que l'époque moderne abandonna complètement l'idée selon laquelle la philosophie est un art de vivre, une quête du souverain bien, bref la recherche du bonheur ici-bas. Et de là émergea une conception plus élevée de la philosophie, dans laquelle celle-ci se voulait indispensable à ce qui a un véritable effet sur le monde, c'est-à-dire la science. Cette mythomanie des philosophes, effet compensatoire de la dépossession successive de leurs objets d'étude initiaux par les sciences de la nature, puis par les sciences humaines, ne fut pas sans effet sur la manière dont ceux-ci abordèrent la lecture des textes de ce qu'il convient maintenant d'appeler d'un sobriquet somme toute assez récent, les grands philosophes. Ce nouveau type de lecture se caractérise par une coupure absolue entre l'action et la théorie, qui atteindra son comble dans la réduction de l'action à un discours sur celle-ci. Le théorique devient par là même auto-suffisant, car il est sa propre fin, et le philosophe se conforte dans le rôle d'homme de cabinet, d'érudit inutile à lui-même et aux autres. Ce faisant, sa réflexion s'égare, guidée par cet aveugle à soi, au monde et aux autres, cet aveugle que Bourdieu nomme « la disposition scolastique $»^{1}$.

Une des conséquences de cette disposition scolastique pour le sujet qui nous intéresse ici, aura été qu'un des aspects sans doute les plus importants de l'ordre géométrique utilisé par Spinoza pour exposer son Ethica, demeura inexploré. Il ne pouvait en être autrement puisque les érudits, ne reconnaissant pas la difficulté propre à l'écriture d'un ouvrage d'éthique, ne pouvaient voir en cet ordre géométrique la solution à cette difficulté.

\section{La question de l'akrasia dans l'Éthique :}

La question de l'akrasia ou de la faiblesse de la volonté est fondamentale pour toute entreprise théorique qui se conçoit comme une éthique. Point nodal entre le théorique et le pratique, ou encore explication que certains qualifieraient de facile pour rendre compte de l'inefficacité du discours théorique en éthique, l'akrasia nous semble néanmoins être une question que toute éthique, ou toute morale, se doit de tenter de résoudre. La solution au problème de l'akrasia doit cependant aller au-delà de l'explication qui en poserait simplement la cause. Sinon, la construction théorique ne resterait

1. Bourdieu, Pierre, Les méditations pascaliennes, Paris, Seuil, 1997, 318 p. 
qu'un discours vide, la création d'un homme de cabinet qui se berce d'illusions en s'enfermant dans un discours inopérant.

Spinoza traite de cette question de l'akrasia dans E4, un lieu qui ne nous étonnera pas puisque cette partie a pour titre De Servitute Humana, seu de Affectuum Viribus ${ }^{2}$. Le premier paragraphe de la Praefatio l'exprime très clairement :

Humanam impotentiam in moderandis, \& coercendis affectibus servitutem voco; homo enim affectibus obnoxius sui juris non est, sed fortunae, in cujus potestate ita est, ut saepe coactus sit, quanquam meliora sibi videat, deteriora tamen sequi. (E4Praef) $)^{3}$

On reconnaît là la formulation traditionnelle du problème de l'akrasia : voir le meilleur et pourtant faire le pire. Spinoza poursuit ce premier paragraphe de la Praefatio de E4 en indiquant que c'est justement la cause de cette chose, et aussi ce qu'ont de bon ou de mauvais les affects, qu'il démontrera dans cette partie.

Toutes les premières propositions de $\mathrm{E} 4$ servent à asseoir la démonstration de la cause de l'akrasia qui trouve une triple formulation dans E4p15, $\mathrm{E} 4 \mathrm{p} 16$ et E4p17. Après avoir démontré dans E4p1 l'irréductibilité de la positivité de l'idée fausse face à la présence du vrai, les propositions 2 à 4 posent la nécessité pour l'homme en tant qu'il est une partie de la nature, d'être toujours soumis aux affects. La cause de la puissance des affects, et les conséquences de cette cause pour l'homme sont développées dans les propositions 5 à 7 . L'importante proposition 8 identifie la connaissance du bien et du mal à la conscience de l'affect de joie et de tristesse, ce qui permettra de poser la cause de l'akrasia à partir de la notion de désir qui fut définie dans la partie précédente comme l'essence même de l'homme, c'est-à-dire l'appétit avec la conscience de l'appétit (E3p9, E3p9S ; E3DefAff1 et son Explicatio ). Le désir étant ce qui détermine l'homme à agir de telle ou telle manière pour assurer sa conservation (E3p9S), avec celui-ci le problème de l'akrasia pourra être posé dans les termes de l'agir. Les propositions 9 à 13 analysent les modalités de la puissance des affects selon que l'image de la chose qui nous affecte est imaginée au sein de la durée, ou comme nécessaire, possible ou contingente.

2. «De la servitude humaine, autrement dit des forces des affects» (p. 335). Toutes les traductions de l'Éthique sont tirées de Spinoza, B., Éthique, Texte original et traduction nouvelle de Bernard Pautrat, Paris, Seuil, 1988, 542 p. Dans la suite du texte la référence à cette traduction sera faite comme suit : $\mathrm{P}$ suivit du numéro du folio. Les références au texte latin sont extraites de l'édition princeps : Spinoza, B., Opera, ed. Carl Gebhardt, 4 vol., Heidelberg, Carl Winters Universitaetsbuchhandlung, 1925. Elle est citée selon la manière usuelle au sein des études spinozistes. Ainsi « G II, 205, 3-5 » signifie le volume 2 de l'édition Gebhardt, page 205, lignes 3 à 5 . Nous avons respecté les italiques de l'édition princeps.

3. "L'impuissance humaine à maitriser et à contrarier les affects, je l'appelle servitude ; en effet, l'homme soumis aux affects est sous l'autorité non de lui-même, mais de la fortune, au pouvoir de laquelle il se trouve à ce point qu'il est souvent forcé quoiqu'il voie le meilleur pour lui-même, de faire pourtant le pire. » (P, 335 ; G II, 205, 7-12) 
La proposition 14 démontre que ce n'est qu'en tant que la vraie connaissance du bien et du mal est considérée comme un affect qu'elle peut contrarier un affect. Cette proposition, qui ne sera pas reprise dans le reste de l'appareil démonstratif de l'Éthique, même si les propositions à partir desquelles elle se démontre le seront (E3DefGenAff, E4p1, E4p7 et E4p8), est importante du point de vue de la problématique de l'akrasia, car celle-ci se pose dans les termes d'une vraie connaissance du bien et du mal, c'est-à-dire d'une connaissance adéquate. Les formulations de E4p15, E4p16 et E4p17 ne laissent pas d'équivoque à ce sujet :

Cupiditas, quae ex vera boni, \& mali cognitione oritur, multis aliis Cupiditatibus, quae ex affectibus, quibus conflictamur, oriuntur, restingui, vel coerceri potest. $(\mathrm{E} 4 \mathrm{p} 15)^{4}$

Cupiditas, quae ex cognitione boni, \& mali, quatenus haec cognitio futurum respicit, oritur, facilius rerum cupiditate, quae in praesentia suaves sunt, coerceri, vel restingui potest. $(\mathrm{E} 4 \mathrm{p} 16)^{5}$

Cupiditas, quae oritur ex vera boni, \& mali cognitione, quaetenus haec circa res contingentes versatur, multo adhuc facilius coerceri potest, Cupiditate rerum, quae praesentes sunt. $(\mathrm{E} 4 \mathrm{p} 17)^{6}$

Dans le Scolie qui suit E4p17 et qui commente le groupe de propositions E4p15 à E4p17, Spinoza, en plus de reprendre la traditionnelle formulation de ce problème - "Video meliora, proboque, deteriora sequor ${ }^{7}$ - donne aussi une formulation qu'il emprunte à l'Ecclésiaste et qui infléchit le sens de la sentence traditionnelle d'une manière qui n'est pas anodine. Cette seconde formulation se lit : "Qui auget scientiam, auget dolorem. " ${ }^{8}$ Si elle n'est pas anodine, c'est qu'elle pose dans toute sa nudité le risque qui pourrait être encouru par une entreprise comme l'Éthique. En effet, si la philosophie spinoziste donnait au lecteur la connaissance de ce qu'est le souverain bien, mais ne lui fournissait pas en même temps les outils nécessaires à l'atteinte de ce souverain bien, alors cette philosophie, selon les principes mêmes exposés dans l'Éthique, ne serait pas cause de joie, mais bien au contraire d'une tristesse, parce que c'est sa pro-

4. «Un désir qui naît de la vrai connaissance du bien et du mal peut être éteint ou contrarié par beaucoup d'autres désirs qui naissent des affects auxquels nous sommes en proie. » (P, 365 ; G II, 220, 2-4)

5. "Un désir qui naît de la connaissance du bien et du mal, en tant que cette connaissance regarde le futur, est plus facile à contrarier ou à éteindre qu'un désir pour les choses qui sont présentement agréables. » (P, 365 ; G II, 220, 24-26) Bien que la proposition ne reprenne pas la formulation « connaissance vraie du bien et du mal », la démonstration elle, reprend cette formule.

6. Un désir qui naît de la vraie connaissance du bien et du mal, en tant qu'elle a trait à des choses contingentes, est encore beaucoup plus facile à contrarier qu'un désir pour les choses qui sont présentes. » (P, 367 ; G II, 221, 6-8)

7. "Je vois le meilleur et l'approuve, je fais le pire. » (P, 367 ; G II, 221, 16-17)

8. «Qui augmente la science, augmente la douleur. » (P, 367 ; G II, 221, 18) 
pre impuissance à atteindre le souverain bien qui serait la plus présente à l'esprit du lecteur. Ce faisant, l'Éthique causerait exactement l'effet inverse de ce qu'elle est supposée faire, nommément nous conduire comme par la main à la connaissance de l'esprit humain et de sa béatitude. Elle serait un livre de la douloureuse conscience de la servitude, c'est-à-dire de l'impuissance, plutôt qu'un texte libérateur.

Soulignons que ce risque est encouru parce que l'Éthique est écrite avec des mots, et qu'elle mobilise donc, de prime abord, une connaissance du premier genre, une connaissance imaginative. Il est donc toujours possible pour le lecteur de lire l'ouvrage et d'acquérir une connaissance seulement imaginative du souverain bien, ce qui, étant donnée la nature inadéquate des idées de ce genre de connaissance, le mettra dans la position d'un Moïse halluciné croyant voir une terre promise qui lui reste interdite.

Si nous écrivons un "Moïse halluciné » c'est que, n'ayant pas une connaissance vraie du bien et du mal, alors bien évidemment ce n'est pas une connaissance vraie qui causerait en lui une tristesse. Car s'il avait une connaissance vraie du bien et du mal, déjà cette connaissance en tant qu'elle est vraie causerait chez lui une augmentation de puissance et donc provoquerait la joie. La formulation spinoziste de la cause de l'akrasia contient donc, comme en germe, sa solution. Et dans cette solution, l'ordre géométrique selon lequel se démontre l'Éthique joue un rôle important, et ce, même si ce rôle n'est probablement pas la seule raison pour laquelle Spinoza a choisi cette forme.

\section{L'akrasia, l'entendement et le lecteur :}

La solution au problème de l'akrasia, solution que nous ne développerons pas ici, trouve son élaboration la plus complète dans E5, car bien que le reste de E4 - de E4p19 à E4p73 et qui pourrait s'intituler « De l'utile et du nuisible à l'homme du point de vue de son essence » — contienne plusieurs règles de vie, autrement dit ce que nous dicte la raison, ces règles seules ne nous indiquent pas comment lutter contre les affects, même si elles nous permettent de voir comme en un tableau ce que serait une vie conduite selon l'essence de l'homme. Elles se servent donc de cet "naturae humanae exemplar " qu'évoque Spinoza dans la préface de cette quatrième partie, et qui doit nous permettre de juger du bien et du mal, du parfait et de l'imparfait dans la sphère de l'action humaine. Ainsi elles nous permettent, selon la formulation traditionnelle du problème de l'akrasia, de voir le meilleur, mais elles ne nous indiquent pas comment éviter le pire.

D'ailleurs quelqu'un qui ne lirait que le résumé qui se trouve en appendice de E4 et qui voudrait suivre ces règles, se trouverait véritablement dans la position de notre Moïse halluciné. Car il voudrait se soumettre à une auto-

9. «modèle de la nature humaine » (P, 341 ; G II, 16 et 19-20) 
rité dont il ne comprendrait pas la nécessité ${ }^{10}$. Et ignorant sa puissance propre d'agir, qui consiste en une détermination de l'agir à partir d'une connaissance adéquate de soi et de Dieu, il pâtirait de son incapacité à suivre ces règles de vie dont Spinoza nous dit qu'elles sont dictées par la raison, mais qui, pour ce lecteur, ne seraient dictées que par Spinoza. C'est peut-être cette difficulté qui amena Spinoza à abandonner le Traité de la réforme de l'entendement. En effet, la forme narrative adoptée pour la rédaction de cet ouvrage et la description, pour ainsi dire extérieure, de l'entendement, ne permet pas au lecteur de faire l'expérience en lui de la raison, de sa puissance et de la joie qu'elle provoque, et ce, même si toutes ces choses sont dites dans le texte. Un monde, véritablement, sépare la lecture de l'expérience de la puissance de l'entendement ( $[\ldots]$ quamdiu mens circa has cogitationes versabatur, tamdiu illa aversabatur, \& serio de novo cogitabat instituto ; quod magno mihi fuit solatio. Nam videbam illa mala non esse talis conditionis, ut remediis nollent cedere. » (TIE, $\mathbb{S} 4)^{11}$ ) de cette expérience effectivement vécue par le lecteur. L'Éthique ne reproduira pas cette erreur du TIE.

La solution au problème de l'akrasia, développée dans E5, a pour clef de voûte une connaissance adéquate des affects. Dans le scolie de la proposition 20 de E5, Spinoza récapitule les remèdes aux affects qu'il a démontrés dans les propositions précédentes, c'est-à-dire tout ce que l'esprit considéré en luimême peut contre les affects. Ces remèdes sont au nombre de 5 , et tous ont à voir avec l'esprit en tant que son essence se définit par l'acte d'intellection :

$I^{\circ}$ In ipsa affectuum cognitione (vide Schol. Prop. 4 hujus). II In eo, quod affectus a cogitatione causae externae, quam confuse imaginamur, separat (vide Prop. 2. Cum eodem Schol. Prop. 4 hujus). III $^{\circ}$ In tempore, quo affectiones, quae ad res, quas intelligimus, referuntur, illas superant, qae ad res referuntur, quas confuse, seu mutilate concipimus (vide Prop. 7 hujus). IV $^{\circ}$ In multitudine causarum, a quibus affectiones, quae ad rerum communes proprietates, vel ad Deum referuntur, foventur (vide Prop. 9 \& 11 bujus). $\mathrm{V}^{\circ}$ Denique in ordine, quo mens suos affectus ordinare, $\&$ invicem concatenare potest (vide Schol. Prop. 10 \& insuper Prop, 1213 \& 14 bujus). ${ }^{12}$

10. On se souviendra que Spinoza écrit, dans une des lettres à Blyenbergh (Ep37), que la connaissance de la nécessité est nécessaire pour l'entendement des fondements de l'éthique.

11. «[...] pendant le temps du moins que mon esprit était occupé de ces pensées, il se détournait des choses périssables et sérieusement pensait à l'institution d'une vie nouvelle; cela me fut d'une grande consolation : le mal, je le voyais, n'était pas d'une nature telle qu'il ne dût céder à aucun remède. " (Spinoza, B., Oeuvres 1 (Court Traité, Traité de la Réforme de l'Entendement, Les Principes de la Philosophie de Descartes, Pensées Métaphysiques), traduction, notices et notes par Ch. Apphun, Paris, Garnier-Flammarion, 1965, p.183 ; G II, 7, 31-34)

12. « (I) dans la connaissance même des affects (voir le Sch. Prop. 4 de cette partie) ; (II) en ce qu'il [l'esprit] sépare les affects d'avec la pensée d'une cause extérieure, que nous imaginons confusément (voir la Prop. 2 avec le même Sch. Prop. 4 de cette partie); (III) dans le temps, grâce auquel les affections qui se rapportent à des choses que nous comprenons l'emportent sur celles qui se rapportent à des choses que nous concevons de manière confuse ou mutilée (voir la Prop. 7 de cette partie) ; (IV) dans le très grand nombres de causes qui alimentent les 
L'ordre même selon lequel sont énumérés ces remèdes marque bien l'importance de celui qui consiste en la connaissance même des affects, qui vient au premier rang. Or les idées adéquates sont les idées de l'entendement, et avant d'avoir une connaissance adéquate des affects, il importe simplement d'avoir une idée vraie, ou plutôt de faire l'expérience de l'idée vraie et donc de l'entendement, ce qui permettra de distinguer l'idée vraie de la fausse, l'imagination de la raison. C'est justement ce en quoi consiste la méthode. Dans Ep37, lettre qu'on pourrait baptiser « la lettre sur la méthode », Spinoza écrit que : «Ex his igitur clare apparet, qualis esse debeat vera methodus, \& in quo potissimum consistat, nempe in sola puri intellectus cognitione, ejusque naturae, \& legam [...] ${ }^{13}$ Et selon E1p31S : « Nihil enim intelligere possumus, quod ad perfectiorem intellectionis cognitionem non conductat. $»^{14}$ Or les démonstrations de l'Éthique - et qui plus est les premières démonstrations du $\mathrm{De} D e o$, qui revêtent une importance plus aiguë pour la question qui nous intéresse - nous font comprendre par l'entendement et nous donnent donc une plus parfaite connaissance de l'intellection.

L'ordre géométrique choisi par Spinoza pour exprimer son Éthique, remplit plusieurs fonctions, mais une qui n'est pas des moindres est justement de faire sentir au lecteur, naturellement et nativement englué dans l'imagination, la puissance de son entendement et sa portée immédiatement éthique. Et de ce point de vue il constitue un élément important dans la solution spinoziste au problème de l'akrasia. L'énigmatique passage de E5p23S, prend alors tout son sens :

At nihilominus sentimus, experimurque, nos aeternos esse. Nam mens non minus res illas sentit, quas intelligendo concipit, quam quas in memoria habet. Mentis enim oculi, quibus res videt, observatque, sunt ipsae demonstrationes. ${ }^{15}$

affections se rapportant aux propriétés communes des choses ou à Dieu (voir les Prop. 9 et 11 de cette partie) ; (V) enfin, dans l'ordre dans lequel l'esprit peut ordonner et enchaîner entre eux ses affects (voir le Sch. Prop. 10 ainsi que les Prop. 12, 13 et 14 de cette partie) » (P, 511 ; G II, 293, 7-17)

13. " On voit par là clairement quelle doit être la méthode et en quoi elle consiste essentiellement, à savoir dans la seule connaissance de l'entendement pur, de sa nature et de ses lois. » (SPINOZA, B., Oeuvres 4 (Traité politique et Lettres), traduction, notices et notes par Ch. Apphun, Paris, Garnier-Flammarion, 1966, p. 251 ; G IV, 188, 19-20 et 189, 1) Il y aurait beaucoup à dire sur ce qu'implique cette définition de la méthode pour la philosophie de Spinoza, à commencer par l'impossibilité d'écrire un Traité de la méthode, c'est-à-dire un livre qui exposerait la connaissance de l'entendement pure, de sa nature et de ses lois. Car les lois de l'entendement sont immanentes à cette activité qu'est l'entendement; pour reprendre une expression de Wittgenstein, nous dirions qu'elles se montrent dans cette activité, et que leur formulation possible serait vide de sens sans la présence en soi de l'expérience effective de l'entendement, et avec cette expérience effective la formulation de ces lois devient superfétatoire. Nous traiterons de cette question de la méthode chez Spinoza dans un prochain article.

14. "Car il n'est rien que nous puissions comprendre par l'intellect, qui ne conduise à une plus parfaite connaissance de l'intellection. » (P, 69 ; G II, 72, 16-17)

15. "Et néanmoins nous sentons et savons d'expérience que nous sommes éternels. Car l'esprit ne sent pas moins les choses qu'il conçoit en comprenant, que celle qu'il a en mémoire. 
Avant d'exposer le fonctionnement de cet ordre géométrique, il importe cependant de rappeler une évidence, qui, probablement à cause de la disposition scolastique de beaucoup de commentateurs contemporains, est sinon oubliée, du moins passée sous silence. Le lecteur de l'Éthique n'est pas le sage, ou même celui qui se croît tel : l'un n'en a guère besoin, et l'autre, par manque d'humilité, est imperméable à toute transformation. Le lecteur de l'Éthique est bien plutôt cet homme - ou cette femme - décrit par Spinoza dans les parties 2, 3 et 4 de son ouvrage. C'est-à-dire un être ayant naturellement certains préjugés - le finalisme, la croyance en une liberté indéterminée, etc. - qui l'empêchent de comprendre l'ordre vrai des choses tel qu'il est. Comme nous le disions précédemment il est englué dans l'imagination. Ce lecteur, c'est bien aussi cet homme qui est évoqué dans le corollaire de la proposition 4 de la quatrième partie que nous avons déjà citée, c'est-à-dire un homme qui "nécessairement, est toujours sujet aux passions, [qui] suit l'ordre commun de la Nature et lui obéit, et [qui] s'y adapte autant que l'exige la nature des choses. » ${ }^{16} \mathrm{Il}$ est aussi cet « homme que mène seulement l'affect ou l'opinion ${ }^{17}$ ce qui, selon le scolie de la proposition 66 de la quatrième partie, a pour conséquence qu'il fait « les choses desquelles il est le plus ignorant ${ }^{18}$. Il est cet homme dont la raison semble impuissante à maîtriser les passions. C'est pour lui que l'Éthique est écrite : c'est justement lui qu'elle mènera " comme par la main à la connaissance de l'esprit humain et de sa suprême béatitude. " ${ }^{19}$ Or si cet homme décrit dans l'Éthique est bien le lecteur de l'Éthique, cette évidence implique que l'Éthique doit être écrite en tenant compte de celui-ci.

Cette constatation, certes banale, est pourtant déterminante car elle nous incite à observer ce que fait effectivement l'ordre géométrique, c'est-àdire l'interaction qu'elle induit entre le texte de Spinoza et le lecteur naif, et non à tenter d'expliquer la forme de l'ouvrage à partir du contexte historique (en la situant au sein de la problématique cartésienne de l'analyse et de la synthèse, ou encore en faisant appel au statut privilégié accordé au savoir mathématique au XVII ${ }^{\mathrm{e}}$ siècle), ou encore à partir des conceptions métaphysiques qu'on retrouve dans l'Éthique (la nature de Dieu comme causa sui et son caractère absolu, ce qui pose son antériorité logique). Nous ne contestons pas la légitimité de ces manières d'expliquer l'ordo geometricus, nous croyons au contraire qu'elles peuvent être tout à fait pertinentes, et que notre explication, loin de les réfuter, pourrait leur donner une vigueur nouvelle.

\footnotetext{
En effet, les yeux de l'esprit, par le moyen desquels il voit les choses et les observe, ce sont les démonstrations elles-mêmes. » (P, 517 ; G II, 296, 3-7) Ici aussi, il y aurait beaucoup à dire sur ces trois petites phrases. Qu'il nous suffise ici de remarquer que dans cette analogie, c'est l'aspect de l'expérience, du sentir, qui se retrouve dans les deux termes qui sont analogues l'un à l'autre.

16. P, 351 ; G II, 213, 31-33.

17. P, 445 ; G II, 260, 23-24.

18. P, 445 : G II, 260, 24-25.

19. P, 93 ; G II, 84, 11-12.
} 
Or comment fonctionne cet ordre géométrique ? Comment cette forme du texte de Spinoza réagit à son lecteur qu'il doit mener à la béatitude, c'està-dire faire passer de l'état de vulgaire à celui de sage ? Nous esquisserons à grands traits les modalités de ce fonctionnement, en insistant surtout sur le tout début de E1, parce qu'il nous semble que celui-ci fait faire au lecteur une expérience éthique fondamentale - expérience déjà décrite dans le début du TIE - et qui sera comme le sol sur lequel le reste de l'Éthique s'appuiera, et à la lumière de laquelle la solution spinoziste au problème de l'akrasia prendra toute sa puissance.

\section{Le début de $\mathrm{E}_{1}$ et l'expérience éthique :}

Le début de l'Éthique est particulièrement aride. L'ouvrage, sans préface ni introduction, s'ouvre sur la définition de la causa sui qui est particulièrement hermétique. Et les choses ne s'améliorent pas au fil de la lecture de ces définitions. La définition de la substance ou celle de l'attribut, celle de la chose libre ou de l'éternité, restent opaques pour le lecteur qui commence la lecture de l'ouvrage. Non seulement il ne comprend rien aux définitions que pourtant il est capable de lire, mais la forme même des définitions lui semble problématique. Toutes, sauf la définition 2 et 7 , ont en effet la forme suivante : par X j'entends (intelligo). L'auteur semble ainsi indiquer qu'il comprend tel ou tel mot de la manière dont il l'a défini. Le lecteur pourrait donc recevoir ces définitions comme de simples définitions nominales, de simples conventions établissant le sens des termes dans la suite de l'ouvrage. Et comme une définition tire sa pertinence de ce qu'elle devrait au moins rendre plus claire la notion définie, le lecteur se trouve ici bien embarrassé.

La lecture des axiomes de cette partie ne nous semble pas présenter ce même genre de difficultés. Ils n'offrent pas la même opacité que les définitions, même si certains sont problématiques. Mais alors pourquoi Spinoza commence-t-il son texte de façon si abrupte et si aride ? La réponse tient dans la cause de l'opacité des définitions.

Le lecteur de l'Éthique, comme nous le supposons, est cet homme décrit dans les parties 2, 3 et 4 de l'Éthique. Or celui-ci s'adapte aux conditions changeantes de son environnement à l'aide d'une connaissance qui est en grande partie une connaissance du premier genre, soit la connaissance par expérience vague et la connaissance à partir des signes, ce genre de connaissance que Spinoza nomme aussi opinion ou imagination. Toutes connaissances tirées des signes sont donc une connaissance du premier genre. Or l'Éthique, comme nous le remarquions précédemment, est, en tant que texte, écrite avec des mots. Conséquemment la connaissance que le lecteur en tirera sera une connaissance du premier genre. Et c'est là que réside la cause de l'opacité des définitions de la première partie de l'Éthique : elles ne possèdent, pour la plupart, aucun contenu imaginatif. Aucune image n'est évoquée par ces signes. Ce faisant, elles ne donnent que très peu de prise au préjugé, mais plus 
important encore, ce début confisque complètement la puissance de l'imagination du lecteur. ${ }^{20}$

Si le lecteur, bien que pour lui les définitions demeurent opaques, poursuit malgré tout sa lecture, une chose assez extraordinaire se produira. C'est qu'en lisant les premières propositions qui suivent les définitions et les axiomes il fera une expérience curieuse et déterminante : il fera l'expérience de l'idée adéquate et ce faisant il éprouvera la puissance de l'entendement. Les démonstrations des toutes premières propositions sont très simples. Ainsi la démonstration de la première proposition (Substantia prior est natura suis affectionibus) dit seulement : "Cela est évident par les définitions 3 et 5 ", et bien que le lecteur ne saisisse pas très bien — ou même pas du tout! — ce qu'est la substance et le mode, la mise en rapport des deux définitions dans la démonstration permet d'affirmer avec certitude que la ou une substance est par nature antérieure à ses affections. Et il en sera de même pour les autres propositions et leurs démonstrations. Le lecteur, petit à petit découvrira diverses relations entre ces notions définies, et ainsi il en appréciera de plus en plus les propriétés au fur et à mesure qu'il les investiguera rationnellement à l'aide des yeux de l'âme que sont les démonstrations.

Mais une autre chose se produira aussi, qui n'est pas moins importante. Il découvrira l'affect lié à la compréhension et à l'augmentation de sa puissance, cet affect que Spinoza nomme la joie. Et la joie ressentie par le lecteur sera d'autant plus forte qu'elle contrastera plus fortement avec le sentiment négatif d'impuissance - de tristesse - que le lecteur aura éprouvé face à la difficulté de comprendre ces opaques définitions.

Cette expérience - celle de la puissance de l'entendement et de la joie qui lui est liée - sera en tant qu'expérience, le terreau sur lequel le reste de l'Éthique pourra s'enraciner ${ }^{21}$. C'est pourquoi si ce terreau n'est pas présent, le lecteur risque fort d'abandonner son effort. Et en ce sens on peut dire que le début de l'Éthique fonctionne comme un filtre à lecteurs. Seront rejetés par le texte ceux qui seront rebutés par l'absence de narration : ceux-là sont rejetés avant même de tenter le voyage. Mais il y a aussi ceux qui, ne portant pas une attention suffisamment grande aux définitions de la première partie -

20. On s'est souvent demandé selon quel genre de connaissance est écrite l'Éthique. La réponse la plus fréquente est qu'elle serait écrite selon le deuxième genre de connaissance. Il nous semble plutôt que l'Éthique n'est pas écrite selon un genre de connaissance ou un autre, mais qu'elle peut-être lue selon un genre de connaissance ou un autre. Et le lecteur qui commence l'Éthique pour la première fois mobilisera assurément le premier genre de connaissance, celui qu'il mobilise le plus couramment.

21. Remarquons au passage que cette manière de faire en sorte que lecteur fasse quelque chose et seulement par la suite d'expliciter dans le corps du texte ce qui a été fait par celui-ci nous semble typique du style de Spinoza. Par exemple, le chapitre méthodologique du TTP est au milieu de l'ouvrage, en un endroit où le lecteur est déjà familier avec la méthode parce qu'il l'a vue à l'œuvre. En paraphrasant la devise cicéronienne, on pourrait dire qu'un des traits stylistiques propres à l'écriture spinoziste, est qu'elle suit la maxime : «Primum facere, deinde explicandi ». 
peut-être est-ce le cas de ceux qui se prétendent déjà sages car ils lisent avec moins d'attention, ne cherchant dans le texte que la confirmation de leur sagesse - et ne remarquant pas que la connaissance qu'ils tirent de la lecture de ces définitions est nulle ou presque, ceux-là aussi seront rejetés ; leur capacité réflexive n'est pas suffisante. Il y a aussi les lecteurs cartésiens, lecteurs qui étaient assurément fort nombreux à l'époque de Spinoza, et qui le sont encore aujourd'hui. C'est un cas particulièrement intéressant. En effet, la terminologie qu'ils découvrent leur étant tout à fait familière, ils croiront comprendre alors qu'en fait ils ne font que plaquer leur savoir cartésien sur un texte qui lui est rétif. Ce n'est qu'au fil de la lecture qu'ils se rendront compte qu'ils font face à quelque chose qui résiste à leur savoir. Ils cesseront peutêtre alors leur lecture, ou encore, pour les plus généreux d'entre eux, ils tenteront de donner une interprétation accommodant la philosophie de Spinoza à la sauce cartésienne, donnant lieu à de multiples difficultés. Et pour les plus érudits des cartésiens, eh bien ceux-là ne manqueront pas de crier à l'hérésie et à la provocation. L'Éthique ne leur semble-t-elle pas écrite selon le style synthétique, style que Descartes réprouvait pour exposer les questions touchant à la métaphysique, car il ne possède pas la force de conviction nécessaire en ces matières, force que seul le style analytique possède, lui qui emporte l'adhésion du lecteur par un exercice de méditation soutenue qui l'amène à la redécouverte des vérités éternelles 22 ? De plus, la première notion qu'ils rencontrent - la causa sui - est cette notion même qui, selon Descartes, est la plus difficile de toute, celle qui ne peut être entendue clairement qu'après avoir parcouru tout l'itinéraire de la via analytica, c'est-à-dire après plusieurs semaines de méditation ${ }^{23}$ ? La tentation de la condamnation de l'Éthique et de son auteur sera alors grande pour ces lecteurs. Tous les autres — s'il en reste ! — seront les lecteurs de l'Éthique.

Cette expérience vécue par le lecteur, qui est une première expérience éthique en tant qu'elle révèle la puissance de l'entendement et la joie qui lui est concomitante, c'est bien l'ordo geometricus qui en est le support. Et elle est importante parce qu'elle a pour fonction d'être le véritable incipit à l'éthique spinoziste. C'est à partir d'elle que la solution au problème de l'akrasia, exposée dans le début de $\mathrm{E} 5$ pourra être entendue comme réellement efficace, parce que la puissance de l'entendement et la joie qui l'accompagne auront déjà été éprouvées par le lecteur.

Mais par delà ce début remarquable de l'Éthique, le reste de l'ouvrage contribue aussi à la préparation du lecteur à la mise en œuvre de la solution du problème de l'akrasia. En effet, le régime de lecture que l'ordre géométri-

22. Voir Descartes, Meditationes de Prima Philosophia, (Responsio ad secundas objectiones) (AT VII 155-159) ; et aussi la traduction française, revue par Descartes et qui diffère sensiblement du texte latin : Descartes, Méditations Métaphysiques, (Réponses aux secondes objections) (AT IX-1 121-123).

23. Voir les réponses au quatrièmes objections faites par Arnauld. 
que impose au lecteur aura pour effet de lui donner cette connaissance de l'entendement pur, de sa nature et de ses lois, en mobilisant constamment la connaissance par l'entendement à l'aide des démonstrations et aussi grâce au système de renvoi qu'elles comprennent. Car s'il est vrai que l'Éthique doit mener son lecteur à la connaissance de l'esprit humain et de sa suprême béatitude, force est de constater que c'est à reculons qu'il nous mène, ce texte! En effet, la forme adoptée par Spinoza pour exprimer sa philosophie contraint le lecteur à toujours revenir en arrière, à relire ce qu'il a déjà lu. Car c'est bien ce que fait le système de renvois mobilisé dans la plupart des démonstrations de l'Éthique. Prenons une proposition au hasard dans l'Éthique, par exemple la démonstration de la troisième proposition de la deuxième partie. Elle renvoie le lecteur à 4 propositions : E2p1, E1p16, E1p35, E1p15. Et ces propositions dans leurs démonstrations renvoient à leur tour à d'autres propositions. Ainsi le lecteur est constamment amené à relire ce qu'il avait déjà lu. Il pourrait sembler que ce type de lecture à reculons est répétitif, mais en fait il n'en est rien. Car chaque démonstration crée un nouveau contexte pour les propositions qu'elle nous amènera à relire. Et dans ce nouveau contexte, c'est très souvent une nouvelle détermination ou implication, une nouvelle puissance des concepts mobilisés dans la proposition relue que découvre le lecteur. Et on pourrait donc dire que, bien qu'il soit amené à revenir en arrière, ce n'est pas à un même texte que le lecteur revient, mais à un nouveau texte puisque, assurément la proposition relue est la même, mais son contexte est différent.

Le fonctionnement de ce système de renvois fait de l'Éthique un livre tout à fait particulier. À la différence d'un livre traditionnel qui n'offre qu'un itinéraire linéaire de lecture, celui des phrases qui s'enchaînent les unes les autres au fil des paragraphes et des chapitres, l'Éthique au contraire est un immense maillage, un réseau complexe fini, qui offre plusieurs parcours au lecteur. ${ }^{24} \mathrm{Il}$ est assez remarquable que Spinoza ait écrit en sous-titre de son Éthique «ordine geometrico demonstrata » et qu'il n'use pas du terme qu'il avait employé pour décrire la forme de son ouvrage sur les Principes de la philosophie de Descartes, c'est-à-dire le terme "mos ». Cette notion d'ordre est en effet capitale à la réalisation du projet de l'Éthique et pour la mise en œuvre de la solution du problème de l'akrasia.

Elle intervient dans plusieurs expressions au sein de l'Éthique, mais pour ce qui nous intéresse les deux suivantes sont particulièrement importantes: l'ordre pour l'imagination et l'ordre pour l'entendement. Dans l'appendice de E1, Spinoza écrit à propos de l'ordre :

Et quia ii, qui rerum naturam non intelligunt, sed res tantummodo imaginantur, nihil de rebus affirmant, \& imaginatiorem pro intellectu capiunt,

24. Observons au passage que le texte de l'Éthique est en fait ce que nous appelons aujourd'hui un texte hypertextualisé. En effet, les renvois dans les démonstrations ne sont rien d'autres que des liens hypertextuels, dont le fonctionnement est identique à ceux que l'on retrouve sur le WEB. Sauf que le réseau de l'Éthique peut être parcouru dans sa totalité. 
ideo ordinem in rebus esse firmiter credunt, rerum, suaeque naturae ignari. Nam cum ita sint dispositae, ut, cum nobis per sensus repraesentantur, eas facile imaginari, \& consequenter earum facile recordari possimus, easdem bene ordinitas, si vero contra, ipsas male ordinitas, sive confusas esse dicimus. ${ }^{25}$

Ceux qui prennent l'imagination pour l'intellect, décrivent donc comme ordonnées les choses qui sont représentées par les sens parce qu'ils ont plus de facilité à les imaginer et à s'en souvenir. L'ordre de ce point de vue n'est donc pas une caractéristique des choses ou de la nature, mais décrit plutôt un certain rapport que nous entretenons avec les choses qui nous sont représentées par les sens; et la différence entre l'ordre et le désordre tient à la facilité ou la difficulté que nous avons à imaginer et à nous souvenir de ces choses. Remarquons que cette caractérisation de l'ordre ne restreint pas l'organisation des relations entre les choses à un type déterminé, ou, ce qui revient au même, à un seul usage du terme ordre. Indifféremment on pourra dire qu'une chambre est en ordre, qu'une série mathématique est ordonnée, ou encore que la représentation anatomique du corps humain est ordonnée. Pourtant dans chaque cas les relations entre les divers éléments sont de type différent.

Mais cette idée selon laquelle l'ordre est lié à la facilité n'est pas exclusive à l'ordre pour l'imagination : on la retrouvera aussi au sein de l'ordre pour $(a d)$ l'entendement. Après avoir démontré dans E5p10D que " aussi longtemps que nous ne sommes pas en proie à des affects qui sont contraires à notre nature, aussi longtemps nous avons le pouvoir d'ordonner et d'enchaîner les affects du corps suivant un ordre pour l'intellect ${ }^{26}$, Spinoza explique dans E5p10S comment, lorsque nous n'avons pas encore une connaissance parfaite - c'est-à-dire achevée — de nos affects nous pouvons faire en sorte de ne pas être affectés facilement (facile) par des affects mauvais. C'est essentiellement grâce à la pratique d'exercices spirituels, que nous pouvons y arriver :

Hac potestate recte ordinandi, \& concatenandi corporis affectiones efficare possumus, ut non facile malis affectibus afficiamur. Nam (per Prop. 7 hujus) major vis requiritur ad affectus, secundum ordinem ad intellectum ordinatos, $\&$ concatenatos coercendum, quam incertos, \& vagos. Optimum igitur, quod efficere possumus, quamdiu nostrorum affectuum perfectam cognitionem non habemus, est rectam vivendi rationem, seu certa vitae dogmata concipere,

25. «Et parce que ceux qui ne comprennent pas la nature des choses, mais se bornent à imaginer les choses, n'affirment rien des choses, et prennent l'imagination pour l'intellect, à cause de cela il croient fermement qu'il y a de l'ordre dans les choses, sans rien savoir de la nature ni des choses ni d'eux-mêmes. Car, quand elles ont été disposées de telle sorte que, lorsqu'elles se représentent à nous par les sens, nous n'avons pas de mal à les imaginer, et par conséquent à nous les rappeler, nous disons qu'elles sont en bon ordre, et, sinon, qu'elles sont en désordre, autrement dit confuses. » (P, 87 ; G II, 81, 36-37 et 82, 1-6)

26. P, 499 ; G II, 287, 4-7. 
eaque memoriae mandare, \& rebus particularibus, in vita frequenter obviis, continuo applicare, ut sic nostra imaginatio late iisdem afficiatur, \& nobis in promptu sint semper. ${ }^{27}$

Ce qui est décrit là n'est ni plus ni moins qu'une restructuration de l'imagination qui s'opère en liant entre elles les images des choses particulières selon un ordre correspondant à l'essence de l'homme, c'est-à-dire un ordre rationnel ${ }^{28}$. Et la pratique de ces exercices fera en sorte que ces images seront plus fortement enchaînées entre elles et que nous les aurons toujours sous la main (in promptu semper). Les exemples que Spinoza donne dans la suite du scolie, font voir qu'ainsi, selon les diverses circonstances de la vie, par cette structuration - cette mise en ordre - de l'imagination selon la raison, il nous sera plus facile d'être moins exposés aux affects négatifs, car la puissance de ce qui les cause, pourra être renversée par la puissance de ce maillage rationnel des images entre elles.

Alors que dire de ce sous-titre " ordo geometrico demonstrata " ? Très sommairement nous pourrions avancer, que la notion d'ordre dans l'Éthique, comme nous venons de le voir, ne doit pas s'entendre comme celui qui serait représenté par celui d'une déduction à partir de principes premiers. L'ordre consiste plutôt en un réseau, un complexe de relations. Et si au premier regard la forme de l'Éthique semble identique à celle de la manière des géomètres, en fait c'est tout autre chose qui se découvre lorsqu'on fait, par une lecture attentive, l'expérience à laquelle cette forme nous convie. C'est à une différenciation, à une complexification croissante de la substance, à laquelle nous assistons au fil des parties de l'Éthique. S'il nous fallait trouver une image pour rendre compte de cette complexification croissante, nous pourrions évoquer celle de l'œuf qui, une fois fécondé, se divise en se complexifiant.

\section{Conclusion :}

Ainsi s'expliquerait donc cette chose si étonnante : qu'un livre d'éthique soit écrit dans une forme qui rappelle celle des ouvrages de géométrie. On voit par là l'importance déterminante que jouent les démonstrations au sein de l'Éthi-

27. « Par ce pouvoir d'ordonner et d'enchaîner correctement les affections du corps nous pouvons faire de n'être pas aisément affectés par des affects mauvais. Car (par la Prop. 7 de cette partie) il est requis plus de force pour contrarier les affects ordonnés et enchaînés suivant un ordre pour l'intellect, que pour en contrarier d'incertains et de vagues. Donc, le mieux que nous pouvons faire, aussi longtemps que nous n'avons pas la connaissance parfaite de nos affects, c'est de concevoir une règle de vie correcte, autrement dit des principes de vie précis, de les graver dans notre mémoire, et de les appliquer sans cesse aux choses particulières qui se rencontrent couramment dans la vie, afin qu'ainsi notre imagination s'en trouve largement affectée, et que nous les ayons toujours sous la main. » (P, 499 ; G II, 287, 20-29)

28. D'où l'on conçoit clairement toute l'utilité de la deuxième partie de E4 et de la construction d'un "naturae humanae exemplar". 
que. Sans elles, point de salut, dirions-nous. Chacune est un véritable exercice spirituel qui prépare le terrain sur lequel les exercices spirituels décrits dans E5p10S pourront se pratiquer, et qui feront en sorte, par une pratique assidue, d'éviter l'akrasia.

On voit aussi par là comment le contenu de l'Éthique a fait retour sur sa forme d'expression et comment celle-ci, loin d'être un vain artifice, une coquetterie d'auteur, joue en fait un rôle déterminant dans l'atteinte du souverain bien ${ }^{29}$.

29. Une première version de ce texte fut prononcée à l'ACFAS en mai 1999. Qu'il me soit permis d'exprimer ici toute ma gratitude envers deux grands professeurs qui m'ont appris à lire Spinoza : MM. Jean-Marie Beyssade et Gilbert Boss. Ce texte leur doit beaucoup, même s'ils se reconnaîtront peu dans cette production d'un élève indiscipliné.

Je remercie les examinateurs de Philosophiques qui ont relu ce texte avec une très grande attention ; leurs remarques me furent d'une grande utilité. Enfin, je remercie Madame Delphine Moreau pour les judicieux commentaires qu'elle m’a généreusement et librement offerts. 